\title{
EDITORIAL
}

\section{PAVING NEW PATHS TOWARD MEDICAL DISCOVERY: THE SECOND GENERATION OF THE MJM}

I am pleased to introduce Volume 2, Number 1 of the McGill Journal of Medicine (MJM). The studies published in this issue of the $M J M$ make significant contributions to the medical sciences, and in several cases promise to uncover new paths toward medical discovery. It is therefore with much respect that I congratulate the student authors on their exceptional research.

One of the greatest attributes of the $M J M$ is its expansive coverage of the medical sciences. This strength is embodied in the current issue, which brings together original student research from such diverse disciplines as pathology, radiology, surgery, and the basic sciences. The first article provides preliminary evidence of the induction of pre-fibrotic liver changes in response to various forms of stress. This study is followed by an investigation revealing the partial purification of a cytosolic kinase involved in a novel regulatory step in triglyceride biosynthesis. In the third article, postoperative utilization of atrial pacing is found to decrease the incidence of atrial fibrillation following coronary artery bypass graft surgery. Using Doppler echocardiography to assess fetal blood flow through the atrioventricular valves, the fourth article reports that well-controlled maternal insulin-dependent diabetes mellitus does not appear to adversely affect fetal diastolic function.

The original research articles published here are complemented by two student-authored reviews. The first of these discusses current theories of pathophysiology in primary open-angle glaucoma and, based upon this knowledge and recently-emphasized patient factors, offers new perspectives on its pharmacological treatment. The second review provides an in-depth analysis of the pharmacological actions and potential clinical applications of the memory-enhancing agent, D-cycloserine.

In the current edition of "Crossroads," the historical development of the relationship between the psychiatrist, the "dangerous patient," and society is traced. In addition, a new feature has been added in this issue of the MJM: a Specialty Profile now complements the Clincial Review and Clinicopathological Correlation of the "MJM Focus" section. This issue's "MJM Focus" is on Cardiothoracic Surgery, and begins with a current profile of specialization in this field. This article is followed by a review of cardiac valve replacement surgery, a complex procedure that in recent years has made crucial advances in extending--and improving the quality of--the lives of millions. The $M J M$ Focus concludes with a report of an unusual presentation of aortic dissection, discovered incidentally during angiographic investigation for suspected coronary artery disease.

With its second volume, the McGill Journal of Medicine remains the only existing medical science journal run by students and publishing original student research from universities around the world. As you likely have discovered in reading previous issues, contributions to the $M J M$ have come from institutions throughout the United States, Canada, and beyond, including France and Ethiopia. Submissions to the forthcoming issue promise to increase the international authorship of the Journal.

Upon publication of the Fall/Winter 1995 issue, the MJM senior staff completed their editorship of the Journal in order to assume full-time clinical responsibilities. Nevertheless, their vision and commitment to the advancement of student research in the medical sciences has established a tradition of excellence that is 
reflected in the Journal held before you. I am honored to serve as the second Editor-in-Chief of the McGill Journal of Medicine, and to be supported by another talented and accomplished Editorial Board.

Clearly, publishing two issues of superior quality per annum can become a full-time job for all those involved, and to do so with such accolades as have been received, while facing the challenges of a demanding medical school curriculum, is a tribute to the dedication of the MJM staff. Each issue is the product of the editorial review process, advertising, solicitation of subscriptions, fundraising, corporate sponsorship, layout, printing, and distribution. No sooner is distribution of the Journal begun than the evaluation of submissions commences for the following issue.

The $M J M$ has made great strides in the brief interim between its second and third issues. An impressive rise in the pool of submissions has not only expanded the Journal's authorship, but has enhanced the rather superb quality of its articles. Furthermore, the $M J M$ has greatly broadened its readership. In addition to circulating to all medical school libraries and select teaching hospitals in Canada and the U.S., the Journal is now shelved by numerous institutions across Europe and Asia. With plans to continue its electronic version of back-issues, the $M J M$ has become one of less than 50 full-text,

on-line medical journals worldwide. We invite you

to visit our site on the World Wide Web at: http://www.medcor.mcgill.ca/MJM/home.html.

I have received numerous requests over the past year from other universities in the United States and Canada, asking how the McGill Journal of Medicine so rapidly progressed from notion to reality, and how these schools can start their own medical journals. A few such journals have recently been initiated, and it is my hope that these and others will advance beyond a local distribution and authorship to one day become, like the $M J M$, an international forum for the communication of medical research by students.

In a recent letter from the Prime Minister of Canada (published at the beginning of this issue), Mr. Chrétien affirms that the impact of the $M J M$ extends far beyond student readership and the shelves of medical school and hospital libraries. What better evidence to support the Prime Minister's viewpoint than the critical commentary of Dr. Peter Black, Neurosurgeon-in-Chief at the Brigham \& Women's and Children's Hospitals at Harvard University, and Chief of Neurosurgical Oncology at the Dana-Farber Cancer Institute. In his communication (published in this issue's Letters to the $M J M$ ), Dr. Black evaluates the previous $M J M$ Focus on Surgery for Temporal Lobe Epilepsy. Undoubtedly, a continuing dialogue between the $M J M$ and its readers is invaluable in shaping the future of this truly unique and exciting journal.

The foundation of the $M J M$ has been built, and its strength tested. Yet, the dynamic interests and involvement of students, physicians, researchers, and faculty are critical to its longevity. On behalf of the Editorial Board, I hope that you share in the tremendous enthusiasm for this issue, and look forward to bringing you new directions in medical discovery with the upcoming Fall 1996 issue of the McGill Journal of Medicine.

Neil A. Goldenberg

Editor-in-Chief

\section{BIOGRAPHY}

Neil A. Goldenberg is the second Editor-in-Chief of the McGill Journal of Medicine. He graduated from Williams College (Williamstown, Massachusetts, USA) in 1993 with a bachelor's degree in English, and is currently in the Class of 1998 at the McGill University Faculty of Medicine (Montreal, Quebec, Canada). In 
the summer of 1990, he served on the editorial staff of Genetic Engineering News (New York, New York, USA). His research experience lies in tumor biology and immunology, including the development of monoclonal antibody conjugates with cytotoxic drugs at the Garden State Cancer Center (Newark, New Jersey, USA), and in vitro studies of antileukemic chemotherapeutics at UMD-New Jersey Medical School (Newark, New Jersey, USA). In 1992 and 1993, he was awarded summer research fellowships at the National Institutes of Health (Bethesda, Maryland) to assist in the development of recombinant viral vaccines in cancer therapy.

Copyright (C) 1996 by MJM 